\title{
A painful periumbilical rash
}

\author{
Sima Amin, BS ${ }^{a}$, Sheevam Shah, MD ${ }^{b}$ (D) J. Scott Thomas, $M^{c}$, and Katherine Fiala, MD ${ }^{a, b}$

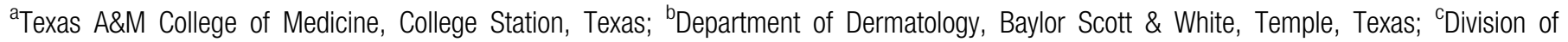 \\ Colon and Rectal Surgery, Department of General Surgery, Baylor Scott \& White, Temple, Texas
}

\begin{abstract}
Jacquet's erosive dermatitis, or dermatitis syphiloides posterosiva, is classified as a severe irritant dermatitis characterized by erythematous papules and nodules with erosive, punched-out lesions. It can be found in the perineal distribution and is frequently reported in infants over 6 months of age. In adults, it is associated with urinary or fecal incontinence. We present a case of a 21-year-old white woman with a history of chronic umbilical drainage and a 3-year history of a periumbilical rash with red, friable, well-demarcated papules. Biopsy revealed pseudoepitheliomatous hyperplasia with focal epidermal erosion and a superficial perivascular infiltrate of lymphocytes and plasma cells. Our case sheds light on this uncommon diagnosis in an unlikely location.
\end{abstract}

KEYWORDS Contact dermatitis; irritant; Jacquet's erosive dermatitis; syphiloides posterosiva

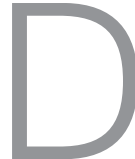

iaper dermatitis is one of the most common dermatologic conditions of infancy. According to the National Ambulatory Medical Care Survey, from 1990 to 1997 there were 8.2 million visits in which diaper dermatitis was diagnosed. ${ }^{1}$ Jacquet's erosive dermatitis, or dermatitis syphiloides posterosiva, is a subset of diaper dermatitis classified as a severe irritant dermatitis. It has become rare in recent years and is associated primarily with urinary incontinence, infrequent diaper changes, or detergent residues in diapers. Clinically, it presents as erythematous papules and nodules with erosive, punched-out lesions. $^{2-4}$ Treatments include avoiding the inciting cause and topical steroids. ${ }^{5}$ We report a unique case of Jacquet's erosive dermatitis associated with chronic umbilical drainage.

\section{CASE REPORT}

A 21-year-old white woman presented with chronic, yellow umbilical drainage and a 3-year history of a pruritic and painful periumbilical rash. She was unresponsive to a 2-week treatment of clotrimazole/betamethasone cream, oral doxycycline, and nystatin powder. Her past medical history was significant for anxiety and depression. Past surgical, social, and family histories were noncontributory. She had one full-term delivery, but the umbilical drainage preceded pregnancy. The patient had no history of piercings. There was no abdominal pain, fevers, chills, recent changes in weight, urinary incontinence, urgency, or hesitancy. Medications included docusate calcium and ferrous sulfate tablets, which were both started during pregnancy.

Surrounding the umbilicus was red, friable, well-demarcated papulonodules within an ovoid yellowish-pink macerated plaque with surrounding atrophy (Figure 1). Serologies for varicella zoster virus, herpes simplex, and rapid plasma reagin were negative. Computed tomography of the abdomen with contrast revealed no significant findings. A punch biopsy revealed psoriasiform dermatitis with focal epidermal erosion and a superficial perivascular infiltrate of lymphocytes and plasma cells. Tissue cultures were negative for fungi and bacteria, but wound culture was positive for actinomyces only in the broth.

The umbilicus was surgically explored to rule out a persistent urachus and revealed a $7-\mathrm{cm}$ defect that tunneled down to the fascia. The wound was irrigated with povidoneiodine solution and packed with iodoform gauze. After surgery, she was treated with wound care and a 1-month course of amoxicillin for the actinomyces grown on wound culture broth. The drainage and erosive lesions initially improved; however, 10 months later, the patient is considering magnetic resonance imaging to further evaluate the soft tissue defect and umbilical excision.

\section{DISCUSSION}

Much of what is known about Jacquet's dermatitis comes from case reports and series. The prevalence of this subset of 


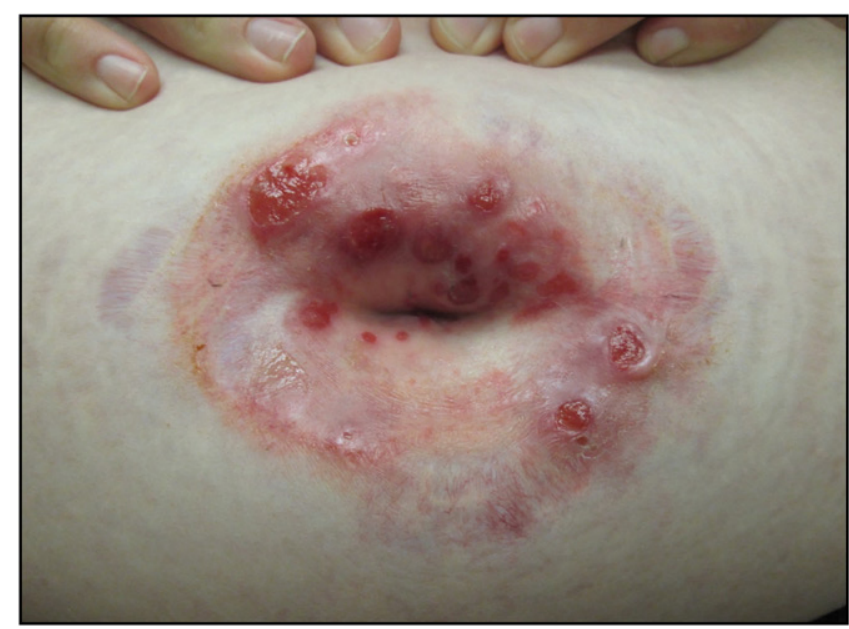

Figure 1. Periumbilical erythema with friable, well-demarcated papulonodules within an ovoid, yellowish-pink, and atrophic plaque.

dermatitis is unknown but is thought to have declined in recent years due to the use of disposable diapers and advances in materials used to make diapers. ${ }^{2-5}$

The pathophysiology of Jacquet's erosive dermatitis is hypothesized to be constant exposure to an irritant, in our case drainage from the umbilicus, causing maceration and an impaired skin barrier. Affected skin is then further predisposed to irritants such as urine, topically applied substances, and regional microflora. Hara et al reported a similar clinical picture of a 9-year-old girl with Jacquet's erosive dermatitis with urinary incontinence. ${ }^{2}$

There is debate about whether Jacquet's dermatitis, granuloma gluteale infantum, and perianal pseudoverrucous papules and nodules are separate entities or are a spectrum of a single condition. ${ }^{6}$ This spectrum of disease most commonly presents in a diaper distribution. It is also imperative to consider syphilis in the differential diagnosis. ${ }^{3-5}$ Grossly, the erosive papules, clinical picture of chronic drainage, and overlying skin irritation, combined with the histological findings of focal epidermal erosion and a superficial lymphocytic and plasma cell perivascular infiltrate, favored a diagnosis of Jacquet's dermatitis in our case.
Treatments for Jacquet's dermatitis include avoiding the inciting cause by keeping the area dry and applying barrier creams and topical steroids. ${ }^{5}$ Rodriguez-Poblador et al reported an 11-month-old girl who was diagnosed with Jacquet's dermatitis in the perianal and perivulvar areas following surgery for Hirschsprung disease resulting in temporary fecal incontinence. ${ }^{4}$ Similar to our patient who was unresponsive to several therapies, their patient was unsuccessfully treated with zinc oxide ointment, burrow solution compresses, miconazole cream, and oral antibiotics. However, resumption of normal stooling cleared the rash. This case highlights the importance of considering Jacquet's erosive dermatitis in areas affected by chronic drainage.

\section{ORCID}

Sheevam Shah (D) http://orcid.org/0000-0002-0838-258X

1. Ward DB, Fleischer AB Jr, Feldman SR, Krowchuk DP. Characterization of diaper dermatitis in the United States. Arch Pediatr Adolesc Med. 2000;154(9):943-945.

2. Hara M, Watanabe M, Tagami H. Jacquet erosive diaper dermatitis in a young girl with urinary incontinence. Pediatr Dermatol. 1991; 8(2):160-161. doi:10.1111/j.1525-1470.1991.tb00309.x.

3. Guerriero C, Paradisi A, Capizzi R, Silveri S. Jacquet papuloerosive dermatitis. J Am Acad Dermatol. 2009;60(3):AB145.

4. Rodriguez-Poblador J, González-Castro U, Herranz-Martínez S, Luelmo-Aguilar J. Jacquet erosive diaper dermatitis after surgery for Hirschsprung disease. Pediatr Dermatol. 2009;15(1):46-47. doi: 10.1111/j.1525-1470.1998.tb01269.x.

5. Paradisi A, Capizzi R, Ghitti F, Lanza-Silveri S, Rendeli C, Guerriero C. Jacquet erosive diaper dermatitis: a therapeutic challenge. Clin Exp Dermatol. 2009;34(7):e385-e386. doi:10.1111/j.1365-2230.2009.03352.x.

6. Robson KJ, Maughan JA, Purcell SD, Petersen MJ, Haefner HK, Lowe L. Erosive papulnodular dermatosis associated with topical benzocaine: a report of two cases and evidence that granuloma gluteale, pseudoverrucous papules, and Jacquet's erosive dermatitis are a disease spectrum. J Am Acad Dermatol. 2006;55(5):74-79. 